\title{
Gait-Based Wi-Fi Signatures for Privacy-Preserving
}

\author{
Yan Li \\ Dept of Computer Science \& Electrical Eng \\ University of Maryland, Baltimore County \\ Johns Hopkins University \\ li@jhu.edu
}

\author{
Ting Zhu \\ Dept of Computer Science \& Electrical Eng \\ University of Maryland Baltimore County \\ zt@umbc.edu
}

\begin{abstract}
With the advent of the Internet of Things (IoT) and big data, high fidelity localization and tracking systems that employ cameras, RFIDs, and attached sensors intrude on personal privacy. However, the benefit of localization information sharing enables trend forecasting and automation. To address this challenge, we introduce Wobly, an attribute based signature (ABS) that measures gait. Wobly passively receives $\mathrm{Wi}-\mathrm{Fi}$ beacons and produces human signatures based on the Doppler Effect and multipath signals without attached devices and out of direct line-of-sight. Because signatures are specific to antenna placement and room configuration and do not require sensor attachments, the identities of the individuals can remain anonymous. However, the gait based signatures are still unique, and thus Wobly is able to track individuals in a building or home. Wobly uses the physical layer channel and the unique human gait as a means of encoding a person's identity. We implemented Wobly on a National Instruments Radio Frequency (RF) test bed. Using a simple naive Bayes classifier, the correct identification rate was $87 \%$ with line-of-sight (LoS) and $77 \%$ with non-line-of-sight (NLoS).
\end{abstract}

\section{Categories and Subject Descriptors}

Biometrics [Cognitive radios]: Privacy-enhancing technology; Cyber-physical security [Intrusion detection]: [RF localization, human signature]

\section{Keywords}

Wi-Fi RF localization, signature, gait, biometrics

\section{INTRODUCTION}

The ability to protect privacy while tracking individuals' locations is critical, especially in public areas, as these locations are bound by laws, restricting image captures or capturing Internet of Things (IoT)'s signals. Clinics that treat diseases, such as Sexually Transmitted Diseases that

Publication rights licensed to ACM. ACM acknowledges that this contribution was authored or co-authored by an employee, contractor or affiliate of the United States government. As such, the Government retains a nonexclusive, royalty-free right to publish or reproduce this article, or to allow others to do so, for Government purposes only.

ASIA CCS '16, May 30-June 03, 2016, Xi'an, China

(C) 2016 Copyright held by the owner/author(s). Publication rights licensed to ACM.

ACM ISBN 978-1-4503-4233-9/16/05 . . \$15.00

DOI: http://dx.doi.org/10.1145/2897845.2897909 carry stigma, cannot capture patients' images or identities. However, big data, which provide trends, contains insightful information. People tracking technology monitors behaviors and develops trust [6].

Traditional human tracking systems are performed by image recognition, thus encountering privacy issues. Floor sensors, wall attachments, and accelerometers are used to measure human activity [34]. Devices like Radio-frequency identification (RFID) [27], door sensors, and ultrasonic sensors [13] were able to track persons. These sensors are intrusive, requiring installation or personal attachment. Microradars [23] utilize special signals and frequency ranges and cause interference. Current tracking techniques require attached devices, such as RFID, smart phones, cameras, or other signal emitting device. Techniques like Hybrid Variable Size Maximum Distance to Average Vector uses microaggregation to protect identity while preserving location [15]. Microaggregation causes loss of location fidelity in order to protect privacy. Access control and encryption limit data availability, requiring authorized users [10].

To solve the problem of intrusion, IoTs process radio frequency (RF) signals with software defined radio (SDR) enabling ubiquitous mobile computations, environment sensing [36] [41] [24] [11], human sensing [37] [2] [3], and movement sensing [21] [26]. With directional antennas, IoTs can improve localization by providing angular information in Wi-Fi networks [31] and improving throughput by directing and localizing energy. In this paper we used angular and polarity features provided by directional horn antennas to detect and identify humans. Wi-Fi standards, used by much of IoT, employ Orthogonal Frequency Division Multiplexing (OFDM) for robustness, allowing the penetration of walls. Currently, Channel State Information (CSI) is used to measure network performance for application layer and to avoid interference. By leveraging CSI, extracted pilot tones and correlated signals, specific features can uniquely identify individuals' gait.

Gait characterization is based on the theory that all organisms utilize central pattern generators (CPG), which produce cyclical patterns produced by the spinal cord utilizing feedback. The study of this phenomenon was first credited to Graham Brown [4] who managed to reproduce stepping patterns without commands from the cortex. Further research showed that special cells from the spinal cord utilized feedback to produce patterns. Because of the unique aspects of human bodies, such as size, leg length, walking cadence, and foot and ankle angles, the signatures generated are currently used in forensics for identification [16]. By measuring 
OFDM signals' multipathings and Doppler shifts, feature sets can identify a person's gait and movement through a hallway and behind a wall by using a fixed antenna and SDR receiver. Wobly passively acquires signal acquisition to form signatures and thus does not interfere or require production of special signals. This human Wi-Fi based signature is a physical attribute-based signature which endorses localization data [20] [39]. In contrast to previous gait and body movement measuring techniques, this technique presented in this paper does not require additional sensors such as privacy intrusive cameras, RFID tags, or ultrasound sensors. In summary, the three fold contributions of this paper are the following:

- Wobly allows for anonymous privacy preserving tracking and positioning based on human gait using Wi-Fi signals. This SDR, biometric, and localization system does not require attachment devices to persons and works in NLoS scenarios.

- Wobly uses standard Wi-Fi beacon signals, works passively to measure Doppler shift and multipathing, and does not require any special signals. The deployment of Wobly followed modern Wi-Fi system architecture.

- Empirical results demonstrated identification of 7 individuals at 3 locations in both LoS (87\% correctness) and NLoS (77\% correctness) scenarios. The results show that the signatures are location and room configuration specific. We implemented Wobly on a National Instrument RF test bed.

\section{MOTIVATION}

This section discusses the need for sensors in different IoT applications, especially smart homes and office, intrusion detection systems, behavior monitoring system, and health care. The information gained from these data enables trend predication, protection from insider threats, and physical area intrusion detection. Embedding such sensors in walls has benefits to security and monitoring, preserving privacy in localization data, and big data for health monitoring (Figure 1). Individual identification can be used in stores, providing customized marketing data or advertisement. Challenges in all these IoT applications include privacy. The signatures in this paper also provide high fidelity localization information, while anonymizing identities, as the signature is unique to sensor locations.

\subsection{Security Applications}

With the increasing need of privacy protection and security from the rise in terrorism, it is critical to develop technologies and sensors to enhance physical security. Physical Intrusion Detection System (IDS) has the ability to tag, track, and identify individuals. Identification and location information are used in access control and behavior monitoring systems. To counter insider-threats, the need to monitor unusual behavior from internal personnel within buildings requires human sensing and localization. Current IDS technology utilizes cameras, RFIDs, IRIS scanners, human voice, infrared, and motion sensors. These sensors are used to protect homes, military and government installations, server farms, and other sensitive locations. By including gait as a hidden biometric, extra layer of restrictions provide addi-

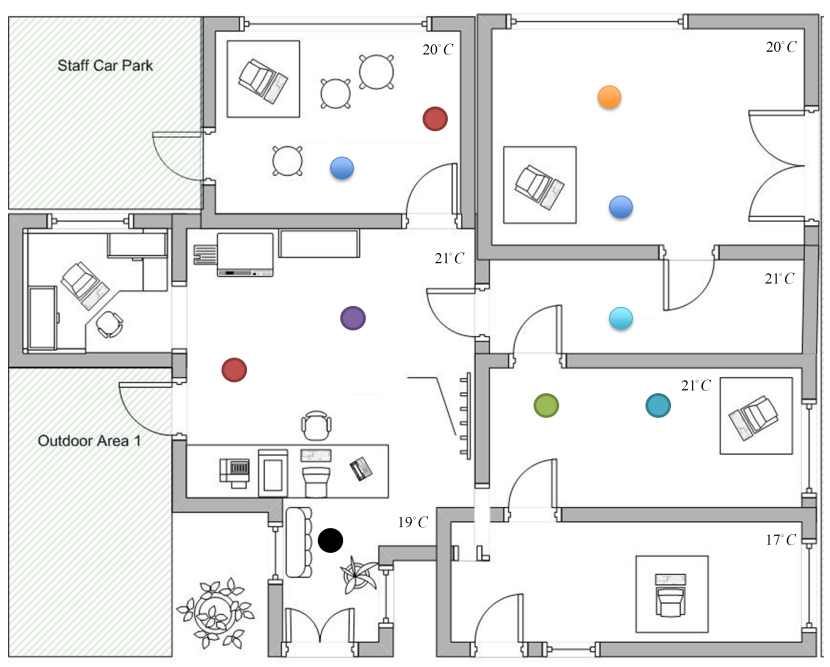

Figure 1: By tracking individuals, smart buildings can enhance security by monitoring behaviors and save energy by customizing temperature and light preferences.

tional security. The benefits of NLoS and standard signals with passive reception allows for clandestine monitoring.

\subsection{Localization Privacy in Smart Buildings}

Preserving privacy in localization and tracking data is critical for smart government facilities, businesses offices, and homes. Tracking individuals in these settings enhances physical security and enable trend forecasting, such as: access control, customized directed marketing techniques, and personalized automation and preferences. Smart buildings that detect the location of individuals can lower energy consumption. People sensing enhances systems like Human Interactions Computer Interactions [14] and Building Operating System Services [8]. However, current techniques require cameras with face recognition algorithms, attached devices, or smart phones, which all contain vulnerabilities. These techniques are intrusive, insecure, and easily violate privacy protection laws. Techniques used for protecting privacy include access control and microaggregation, which limits accuracy and access to information. The high fidelity localization signatures discussed in this paper provide anonymization using physical layer security, by encoding human gait and physical channels.

\subsection{Preserving Privacy in Healthcare}

Current use of accelerometers and attached devices such as smart phones allows for big data collection to detect trends in healthcare. With additional features, such as location and movement speed, these trends can provide insight on disease progression and causes (Figure 2). Storing such information in a health database helps predict trends and diagnosis. Monitoring the elderly and young individuals benefit healthcare automation, and thus lower costs. The data gathered from clinics are sensitive, as diseases such as HIV carry stigma. Current technologies protect this data by access control, thus limiting data availability. These technologies contain vulnerabilities and may violate privacy laws. With the ability to penetrate walls and anonymiza- 
tion, Wobly enables human sensing without attached equipment and raises fewer privacy concerns.

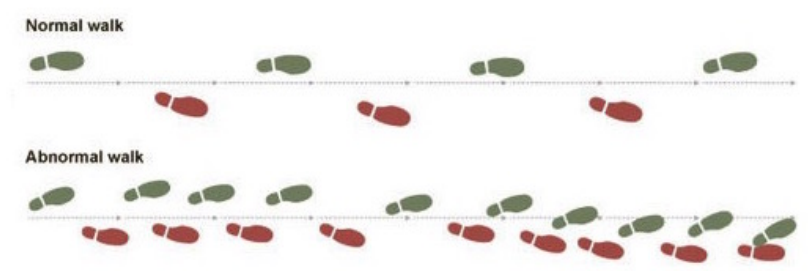

Source: Erasmus MC Medical Center in Rotterdam

Figure 2: Identify changes in gait caused by neurological disease [18]

\section{THREAT MODEL}

The threat that typically occurs with tracking systems is that adversaries may access the location histories of the targets. Attackers may gain access to location tracking services, camera recording systems, or targets' IoTs such as smart phones and wearables [5]. The access means are typically software code fallacies, eavesdropping, socially engineered techniques, or identity theft. The users of Wobly trust the facility and believe that no other identity recording systems are active.

Our assumptions are that adversaries, who may have access to signatures, do not have access to the room configuration and the targets simultaneously. For privacy, we also assume that identities are not associated with the signatures in any manner that exposes the sensitive information.

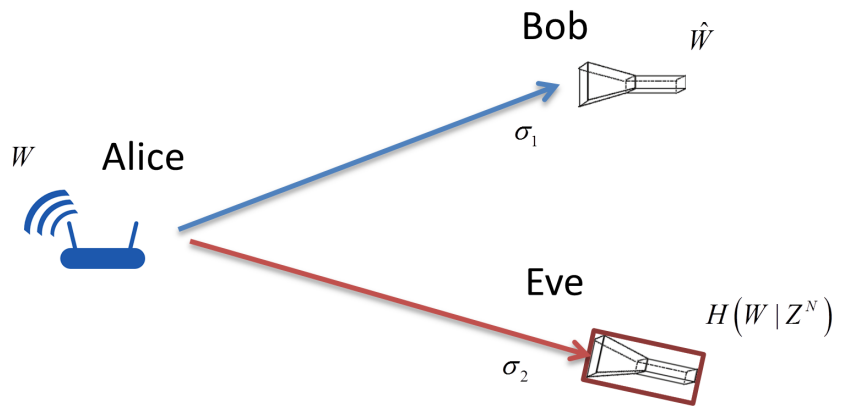

Figure 3: Wyner described a wiretap channel, showing Alice communicating with Bob, with Eve as the eavesdropper.

Because Wobly depends on physical layer channel encryption, we can utilize Wyner, Cheong, and Hellma's Wire-Tap Channel model (Figure 3) [19]. Wyner relaxed Shannon's model by adding noise to transmitted channel. In Wyner's wiretap modem, $W$ is the message sent, $\hat{W}$ is the message received, and $Z$ is the entropy of the message. Wyner defined the equivocation or the confusion in Equation 1 and perfect secrecy in Equation 2 for $n$ channels.

$$
\begin{gathered}
\frac{1}{n} H\left(W \mid Z^{n}\right) \\
H\left(W \mid Z^{n}\right) \approx H(W)
\end{gathered}
$$

Cheong and Hellma apply a statistical description of the channel and define channel secrecy in Equation 3.

$$
C_{s}=\frac{1}{2} \log \left(1+\frac{P}{\sigma_{1}^{2}}\right)-\frac{1}{2} \log \left(1+\frac{P}{\sigma_{1}^{2}+\sigma_{2}^{2}}\right)
$$

Where $\sigma_{1}$ and $\sigma_{2}$ are receiver and eavesdropper channels, and $P$ is the power level of the signature. Applying this to Wobly, Wyner theory suggests that the more the adversary's channel is different than the receiver's channel, the more secret Wobly will be. Therefore, we assume that the there are no adversaries' antennas placed close to Wobly's antenna.

\section{SYSTEM ARCHITECTURE}

This section presents a design solution to several challenges in real-world Wi-Fi deployments and signal acquisition. Section 4.1 discusses the RF test bed architecture including antenna design for acquiring and processing the $\mathrm{RF}$ signal. Section 4.2 shows the production of the $\mathrm{Wi}-\mathrm{Fi}$ beacon. Sections 4.3 discusses the Doppler and multipath channel encoding caused by human gait and room configurations. Section 4.4 explores the receiver and signal processing. Sections 4.5 and 4.6 demonstrate techniques to measure Doppler and multipath effects forming signatures, and section 4.7 discusses using wavelets to characterize signatures.

Challenges include 1) obtaining RF signals with high SNR in homes and buildings; 2) designing the SDR system using standard modern Wi-Fi system deployments; and 3) mitigating high possibility of interference in the open ISM band. To simulate modern day RF in buildings, RF over optical fiber and standard preamplifier were placed as close to the antenna as possible (shown in Figure 5), providing high SNR at great distances. The acquisition system operated within the $2.4 \mathrm{GHz}$ ISM band, using only OFDM and Binary Phase-shift Keying (BPSK) modulation, meeting the 802.11X specification for beacons. To account for IoT devices, which may interfere, a highly directional high gain ARA horn antenna obtained the signal. Cross-correlation, which amplifies a high specific signal, also mitigated thermal and other interference.

\subsection{System Design}

In this section, challenges include mimicking a transmit Wi-Fi router using 802.11 standards and storing the signals. The NI RF test bed must stream the received signals for an entire duration in which persons stroll through a room and hallway. The horn antenna has the ability to discriminate between horizontal and vertically polarized signals. In this experiment, by measuring the horizontal component of the electromagnetic field, the antenna is able to sense shifts from the vertical component. Thus when a vertical component becomes horizontal due to multipathing, the electromagnetic waves' horizontal component suddenly increases.

A transmitter (TX) and receiver (RX) pair operated on the same NI PXIE system. The TX generated a pulsing OFDM signal at $1 / 3$ duty cycle simulating a Wi-Fi beacon signal using an omni-direction (shown in Figure 4). The RX digitized the baseband signal, streaming the oversampled digitized values to the redundant array of independent disks (RAID) drive. Processing the stream value, an algorithm demodulated the OFDM signal and recovered the pilot tone. The received pilot tones were compared to the transmitted values, and the Doppler signature was computed. The re- 


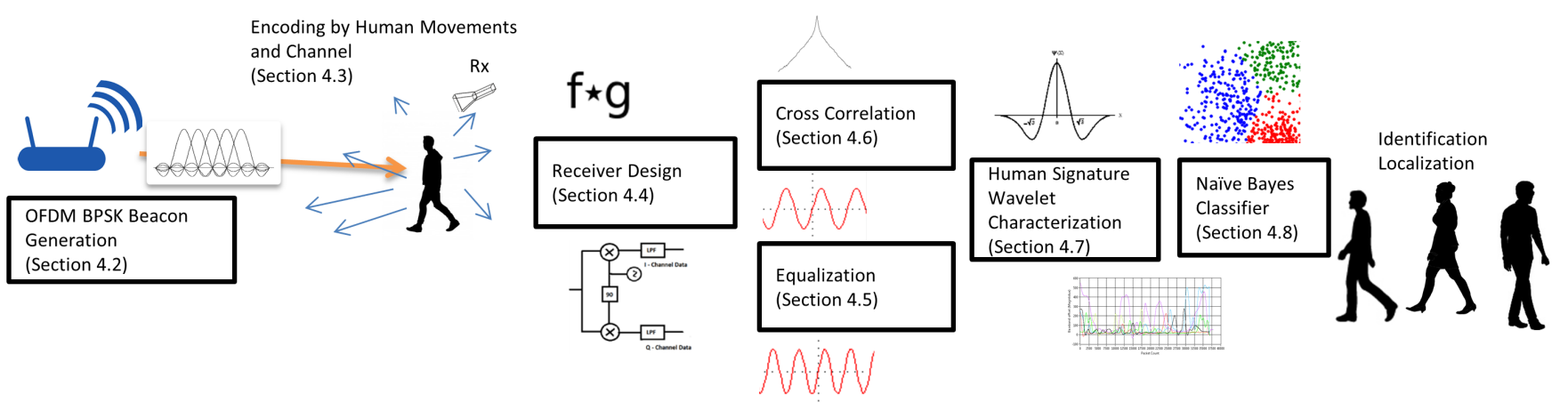

Figure 4: Overall SDR System Architecture consists of TX sending a pulsing BPSK OFDM modulated signal and Rx demodulating the signal.

ceived packets were correlated against the transmitted packets to produce the multipathing signature.

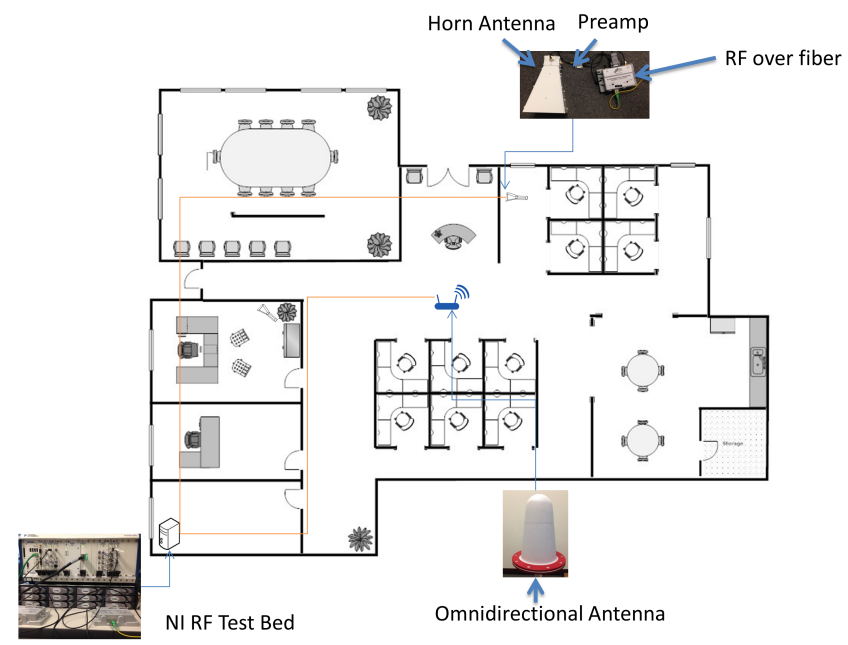

Figure 5: Horn antenna, preamp, and RF over fiber capture RF signals, provided directionality, and increased SNR in a real world deployment. The NI RF test bed consists of signal acquisition (PXIe-5622), signal generation (PXIe-5652), down-converter (PXIe-5601), and up-converter (PXIe-5450).

\subsection{OFDM Signal Design}

This section discusses the theories of generating a $\mathrm{Wi}$ Fi beacon, which uses an OFDM BPSK modulation and a cyclic prefix. The challenges addressed in this section include simulating a Wi-Fi beacon with CSI estimating pilot tones and generating the ISM band signal. An OFDM signal is created by turning binary data into cyclical representations, usually taking the form of frequency, amplitude, or phase. In 802.11 standards for Wi-Fi beacons, this representation is Binary Phase Shift Key (BPSK). BPSK has two states representing 0 and 1 .

$$
S_{n}(t)=\sqrt{\frac{2 E_{b}}{T_{b}}} \cos \left(2 \pi f_{c} t+\pi(1-n)\right), n=0,1 .
$$

Where $f_{c}$ is the center frequency. $E_{b}$ and $T_{b}$ are constants defining energy and duration per bit respectively. To minimize inter-symbol interference, the BPSK signal contained a cyclic prefix. The prefix is added to the beginning of the sig- nal by copying portions of the end signal. This prefix added signal is the definition of a symbol, representing binary data.

$$
s_{n}^{\prime}[t-N]=\left[s_{n}[N-L+1] \ldots s_{n}[0] \ldots s_{n}[N-1]\right]
$$

Where $L$ is the length of the prefix.

The reason why OFDM is such a popular encoding scheme is that multiple bits can be sent simultaneously (Figure 6) using multiple sub-carriers. With guard intervals, cyclic prefix, rate control, and equalization schemes these signals are resilient against Doppler, multipathing, and fading interference. To simulate a beacon, the signal consisted of $20 \mathrm{MHz}$ of bandwidth with 51 active subcarriers. The 51 carriers, derived from 51 bits, were summed together by the parallel to serial converter. OFDM utilizes the inverse Fast Fourier Transform (iFFT) to allow the subcarriers to be orthogonal, allowing for high spectral efficiency (shown in Figure 6 ). Modern communication systems define specific subcarriers as pilot tones, sent to perform channel estimation. To simulate this scheme, the NI system used an arbitrary wave generator (AWG) at baseband, up-converting the signal to $2.412 \mathrm{GHz}$, and sending pulsed OFDM signals at 1/3 duty cycle. This up-conversion process centers the baseband signal (Figure 7) to the desired transmit frequency by first up-sampling, then multiplying with a desired frequency sine wave, and finally high-pass filtering.

\subsection{Encoding Mechanism: Doppler Shift and Multipath}

This section discusses the Doppler channel and multipath encoding an electromagnetic wave caused by individuals' attributes when moving. The challenge addressed in this section is modelling Doppler Effect and multipath based on human gait. The human body moves about 1.4 meters per second, which translates to an approximate $11 \mathrm{~Hz}$ shift based on the Doppler Effect. Other papers, however, have demonstrated that movements of legs, arms, and feet happen much faster, at around $200-300 \mathrm{~Hz}$, yielding about 20-40 meters per second [23]. The shift occurs from the electromagnetic waves reflecting off of a moving body (shown in Figure 8). Incorporating the three frequency components from arm, leg, body, and original signal yields Equation 6 .

$$
f_{D}=\frac{\Delta v_{1}}{c} f_{1}+\frac{\Delta v_{2}}{c} f_{1}+f_{1}
$$

After extracting the components of the velocity vector, the equation simplifies to Equation 7: 


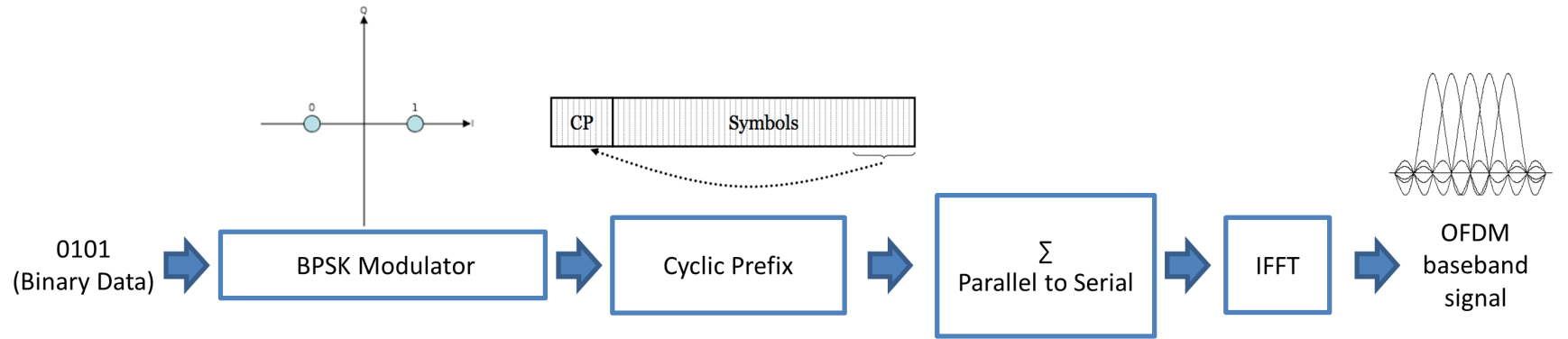

Figure 6: Baseband OFDM BPSK modulated signal allows for multiple transmissions of multiple bits simultaneously.

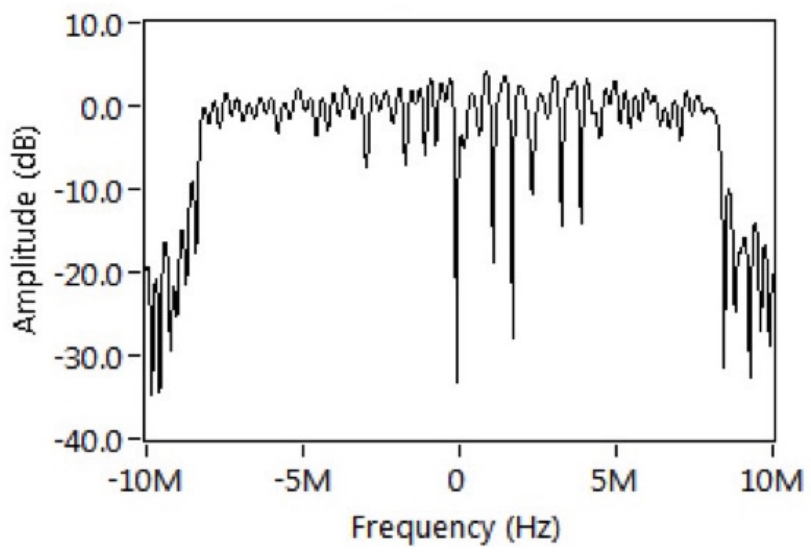

Figure 7: Baseband OFDM BPSK Modulated Signal Beacon Transmitted

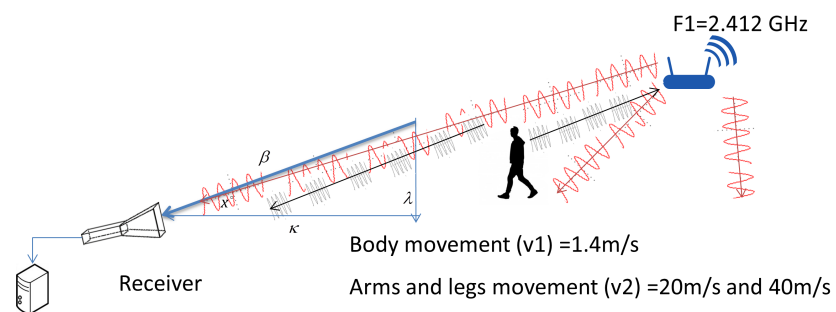

Figure 8: Because of the moving person, the reflected signal contained frequency shifts (Doppler Effect). Both the velocity vector and the Doppler shift could be broken into the vertical and horizontal components.

$$
f_{D}=\frac{\beta}{c} f_{1}\left(\Delta v_{1}+\Delta v_{2}\right)\left(\sin \left(x^{\circ}\right)+\cos \left(x^{\circ}\right)\right)+f_{1} .
$$

When electromagnetic waves propagate and encounter an object, the waves' reflections and refractions cause different times of arrivals (Figure 9). The signals' polarities may also change, and the delayed signals' powers will be significantly less. Multipath is defined in Equation 8:

$$
\sum \rho_{k} e^{j 2 \pi f_{D} k} \delta\left(t-\tau_{k}\right)
$$

Where $\rho_{k}$ represents power lost during each delay, $e^{j \phi k}$ represents the signal, and $\delta\left(t-\tau_{k}\right)$ impulse function represents the delay.

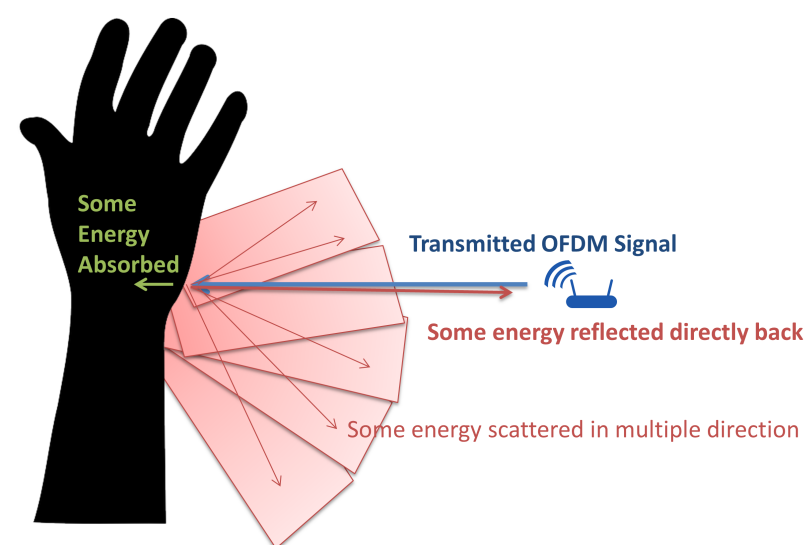

Figure 9: When an electromagnetic wave encounters an object, some energy is absorbed, reflected back, and scatter in different directions.

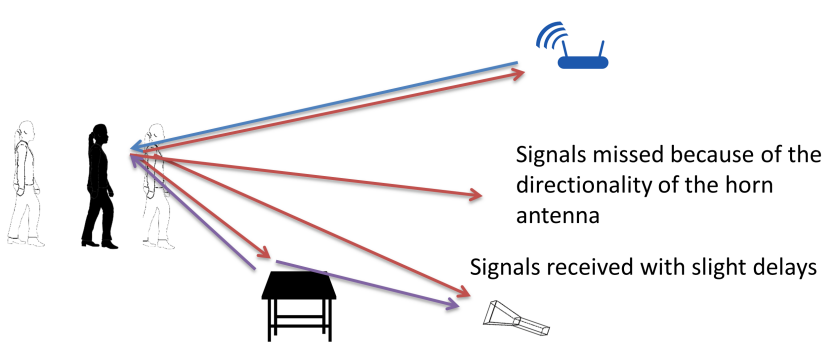

Figure 10: As humans and room configuration change, the multipath signals also change. Because human gait is relatively consistent, we can encode a human signature using the physical channel, sensed by Wobly.

As the individuals move or room configurations change, the multipaths and Doppler shifts causes the physical channel to change (Figure 10). By sensing those changes, the system produces the human signature.

\subsection{Receiver Design and Signal Processing}

This section discusses converting a high frequency with Doppler and multipath interference (discussed in section 4.3) into a usable baseband signal. First, the signal was conditioned by down-converting and down-sampling from 2.412 $\mathrm{GHz}$. Then, a low pass filter removes the upper side band signal, which is an upper frequency duplication. These fil- 
ters are typically a digital cascaded integrator comb finite impulse response filter.

$$
A_{R} \cos \left(2 \pi f_{D} t+\phi_{D}\right) A_{L O} \cos \left(2 \pi f_{L O} t+\phi_{L O}\right)
$$

Applying the local oscillator frequency, $f_{L O}$, and the received signal frequency, $f_{R}$, signal yields Equation 10 .

$$
=\frac{1}{2} A_{L O} A_{R}\left(\begin{array}{c}
\cos \left(2 \pi\left(f_{D}-f_{L O}\right) t+\left(\phi_{D}-\phi_{L O}\right)\right) \\
+\cos \left(2 \pi\left(f_{D}+f_{L O}\right) t+\left(\phi_{D}+\phi_{L O}\right)\right)
\end{array}\right)
$$

Low pass filter removes the upper side band signal, which is an upper frequency duplication (shown in Equation 11).

$$
\frac{1}{2} A_{L O} A_{R} \cos \left(2 \pi\left(f_{D}-f_{L O}\right) t+\left(\phi_{D}-\phi_{L O}\right)\right)
$$

Applying Doppler channel effect yields the Equation 12.

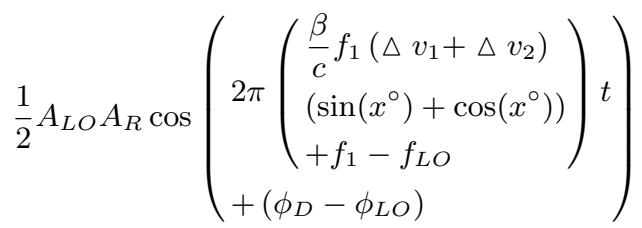

Because the NI system receiver and transmitter used the same clock, the phase difference would be zero (Equation 13), and there would not be any difference in the received frequency and the oscillator frequency, apart from Doppler shifts (Equation 14). Because the receiver signal's frequency $\left(f_{R}\right)$ and the local oscillator $\left(f_{L O}\right)$ operate on the same frequency, the processing operated on the baseband signal (defined in Equation 15).

$$
\begin{gathered}
\phi_{D}-\phi_{L O}=0 \\
f_{1}-f_{L O}=0
\end{gathered}
$$

Letting $\kappa$ be the combined constants and removing the cancelled variable yields the baseband (Equation 15).

$$
B r(t)=\kappa \cos \left(2 \pi\left(\begin{array}{c}
\frac{\beta}{c} f_{c}\left(\Delta v_{1}+\Delta v_{2}\right) \\
\left(\sin \left(x^{\circ}\right)+\cos \left(x^{\circ}\right)\right)
\end{array}\right) t\right)
$$

Thus down-conversion removes the carrier frequency from the Doppler shifted signal in the baseband signal.

\subsection{Utilizing OFDM equalization}

This section discusses using the theory of OFDM BPSK demodulation to recover the pilot tones and determine equalization values. These equalization values measure the channel interference discussed in section 4.3 to form the first feature set. Equalization schemes in modern day communication standards measure Doppler Effect. The equalization scheme operates on the pilot tones sent to perform channel estimation. To obtain the pilot tone levels, an OFDM demodulator must be implemented. Because OFDM is a linear operation, demodulation reverses the modulation operations using a Fast Fourier Transform (FFT). By subtracting the expected and received pilot tones frequency offset over time, this measurement can provide the Doppler frequency shift in the signature (shown in Equation 16) (Figure 11).

$$
S_{1}=\sum_{k} \rho_{k} \kappa \sin \left(\pi f_{D}+f_{c}\right) \sin \left(\pi f_{D}-f_{c}\right) \delta\left(t-\tau_{k}\right)
$$

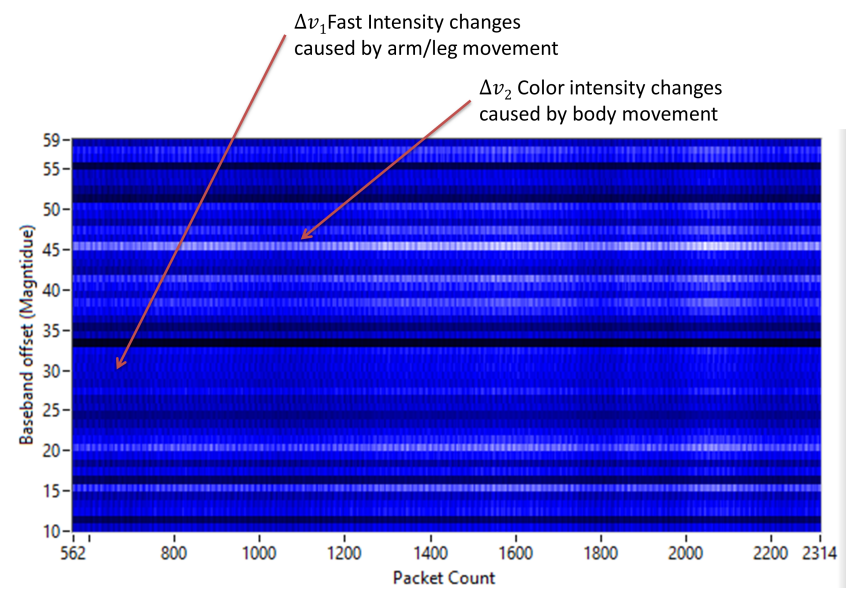

Figure 11: This heat chart shows received pilot tones offsets with Doppler Effect caused by arms, legs, and body movements.

The signatures were created by measuring the area of peaks of $S_{k}$ by using a wavelet transform and coefficients to find valleys and integrating the peaks of all the $S_{k}$ [1]. Let $S_{k}\left(j_{m}\right)$ be the $m^{t h}$ local minimum (described in section 4.7). For $n$ local minimums, the feature vector can be defined in Equation 17.

$$
f(j)=\left[\sum_{j=m}^{m+1} S_{k}(j), \sum_{j=m+1}^{m+2} S_{k}(j) \ldots \sum_{j=m+n-1}^{m+n} S_{k}(j)\right]
$$

\subsection{Utilizing Cross Correlation}

This section discusses the theory of cross-correlation to measure multipathing in order to define the second feature. The premise of correlation is to measure two signals' similar-

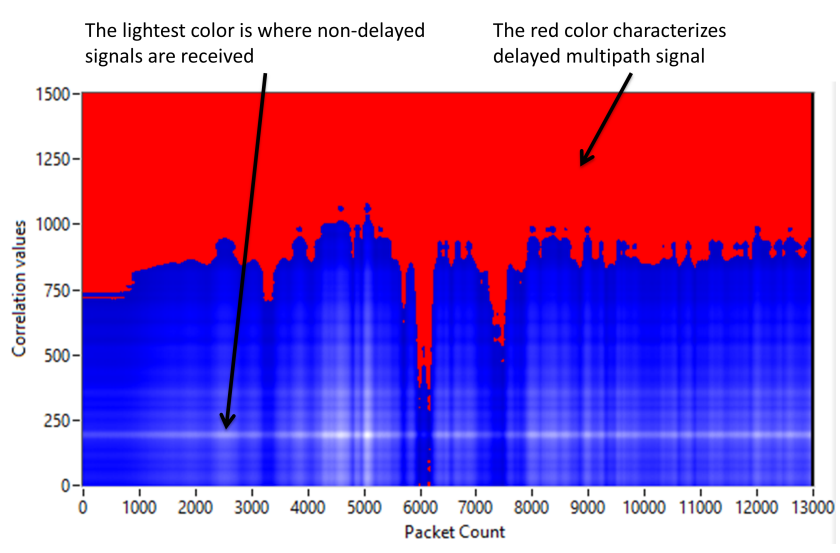

Figure 12: This heat chart shows the correlation between sent and received signals demonstrating multipath.

ities. This computation amplifies the desired signal and suppresses noise. By multiplying the received and sent signals, the closer the match, the higher the output would be (Figure 13). By performing this multiplication on the time domain signals and spanning it over time, the algorithm could detect the multipath signals and remove noise interference (Figure 12). 
Let $x(k)$ be the sent signal, $y(t)$ be the received pulse signal, and $\eta(t)$ a Gaussian be the noise model of electromagnetic interference thermal noise. The time domain correlation is defined in Equation 18.

$$
\sum_{t} b^{*}(k) B r(k+t)+b^{*}(k) \eta(t)
$$

Because the non-correlative nature of the thermal noise, we can assume $x^{*}(k) \eta(t)$ to be sufficiently small, and thus it can be removed. Based on the values of the correlator function,

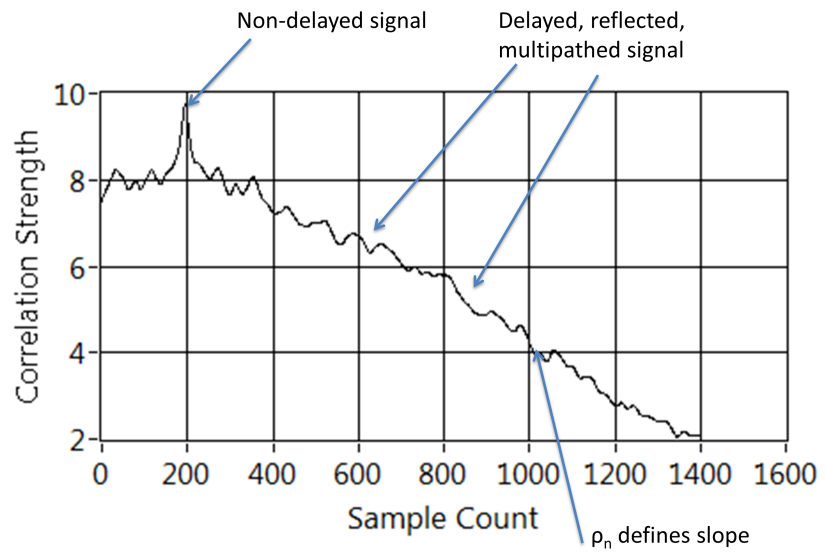

Figure 13: Correlation between a received noisy OFDM burst and the original signal shows the time delayed, multipathed signals.

a heuristic value of half the peak correlation describes the signature. Therefore, let $M_{\max }$ be the max correlation value and $M_{\max / 2}$ be the correlation value half of the max amount. Let $\max$ and $\max _{2}$ be the index associated with those values respectively. Therefore we can define the distance of the correlation to be $S_{2}(i)=\max _{2}-\max$. The same processing method defined in Equation 17 produced the second feature vector.

\subsection{Wavelets Characterization of Signatures}

This section describes use of wavelet transforms, which characterizes signature by creating feature vector used in classifiers. The continuous wavelet transforms (CWT) sum orthonormal series, generated by wavelets, to represent the original signal (defined in Equation 19) [9].

$$
\left.C(a, b)=\int_{R} s(t) \Psi_{a, b}\right)(t)=\frac{1}{\sqrt{a}} \psi\left(\frac{1-b}{a}\right)
$$

Where $a$ is wavelet's scale. $b$ is the wavelet's translation, $\psi$ is the mother wavelet, and $\Psi_{a, b}$ is the scaled and translated wavelet. The wavelet transform is similar to the Fourier transform, which sums Sine waves. Suppose that the local minimum of the signal is defined as $M_{\text {sig }}$. We can define Equation 20 as the signature Equation.

$$
M_{\text {sig }}(t)=M(t)+B(t)+C, t \in\left(t_{1}, t_{2}\right)
$$

Where $M(t)$ is the minimum; $B(t)$ is the baseline function with zero mean; $C$ is a constant; $t$ defines local minimum in the region of $t_{1}$ and $t_{2}$.
CWT's coefficients of the minimum are defined in Equation 21.

$$
\int_{R} M(t) \Psi_{a, b}(t) d t+\int_{R} B(t) \Psi_{a, b}(t) d t+C \int_{R} \Psi_{a, b}(t) d t
$$

Where $M(t)$ is the minimum; $B(t)$ is the baseline function with zero mean; $C$ is a constant; $t$ defines local minimum in region of $t_{1}$ and $t_{2} . \quad a$ is wavelet's scale; $b$ is the wavelet's translation; $\psi$ is the mother wavelet; $\Psi_{a, b}$ is the scaled and translated wavelet. The local minimums of the signatures used the standard Mexican Hat wavelet for the mother wavelet for symmetry. Because the wavelet has a zero mean and the Mexican Hat wavelet is symmetrical (Equation 22), the second and last term can be removed. Equation 21 is left with just the minimum points.

$$
C \int_{R} \Psi_{a, b}=0 \int_{R} B(t) \Psi_{a, b}(t)=0
$$

\subsection{Naïve Bayes Classifier}

This section describes using a naïve Bayes classifier to distinguish between persons using the feature from Section 4.7. First the data was filtered. The filter was a simple threshold that removed items from the array that were close to baseline cases. The feature points that fell outside the $10 \%$ standard distribution were also removed, as they were artifacts caused by noise. The naïve Bayes used two fold cross validation upon the feature sets.

Let classes be $\mathrm{k}$ individuals $\left(A_{k}\right)$; cross correlation $\left(f_{c c}(k)\right)$, pilot tone $\left(f_{p t}(k)\right)$, and packet count $\left(f_{\left(p_{-} c n t\right)}(k)\right)$ are the observed features. Thus the naive Bayes classifier is defined in Equation 23, selecting the largest and closest probability given the observations.

$$
\underset{k \in\{1, \ldots, k\}}{\arg \max } p\left(A_{k}\right) p\left(f_{c c}(k) \mid A_{k}\right) p\left(f_{p t}(k) \mid A_{k}\right) p\left(f_{\left(p_{-} c n t\right)} \mid A_{k}\right)
$$

\section{PERFORMANCE EVALUATION}

This section discusses the empirical implementation to measure the two features. The challenge addressed is setting up a repeatable realistic test environment, producing a visualization demonstrating differentiation, and then producing a statistical description of differentiation. Section 5.1 discusses the control or the baseline cases, showing noise rejection. Section 5.2 shows the 12 signatures obtained from the experiment. Section 5.3 discusses location and multipathed physical layer channel encoding using empirical data. Section 5.4 demonstrates the signature as distinguishable.

To simulate a clinic in which individuals with different age ranges and sexes visited, we selected seven different individuals. The individuals selected were three males and three females. Their ages range from teens to 60s. Depending on the individual, the entire walk path lasted between 713 seconds. Based on the packet count, recorded time, and start location, the location of the individual was recorded throughout the experiment.

Seven individuals walked in the middle hallway and in a room for about five meters, repeating this action several times. For the NLoS case, the horn antenna was placed in a different room, 0.5 meters away from the wall, while 


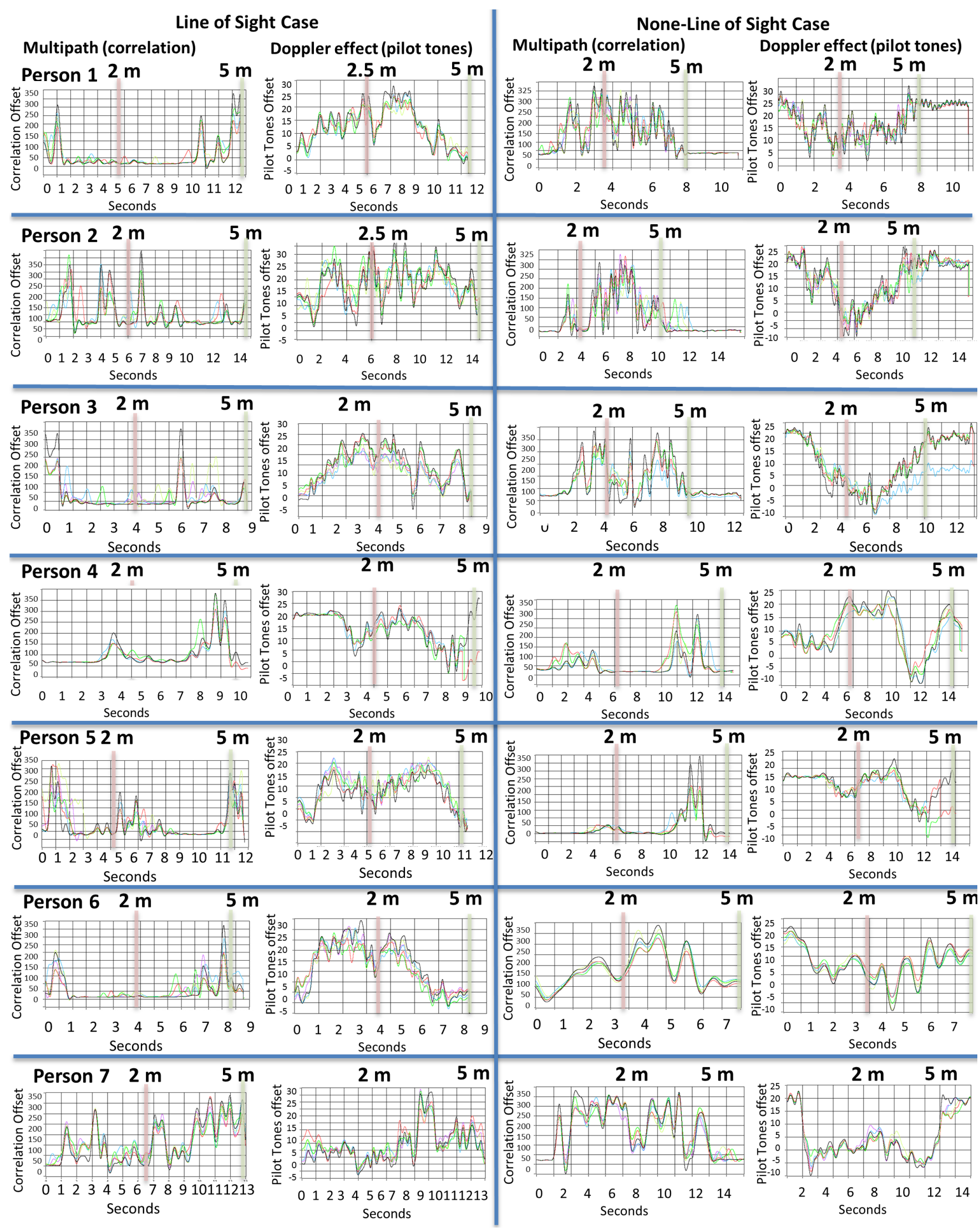

Figure 14: Human signatures of seven individuals walked repeatedly multiple times (shown in colors) demonstrated stability and uniqueness. Based on elapsed time, location markers for 2 and 5 meters (shown in red and green highlights) demonstrate localization. Person 1 was male, age 29. Person 2 was female, age 26. Person 3 was male, age 49 . Person 4 was female, age 59. Person 5 was male, age 19. Person 6 was female, age 15. Person 7 was male, age 21. 


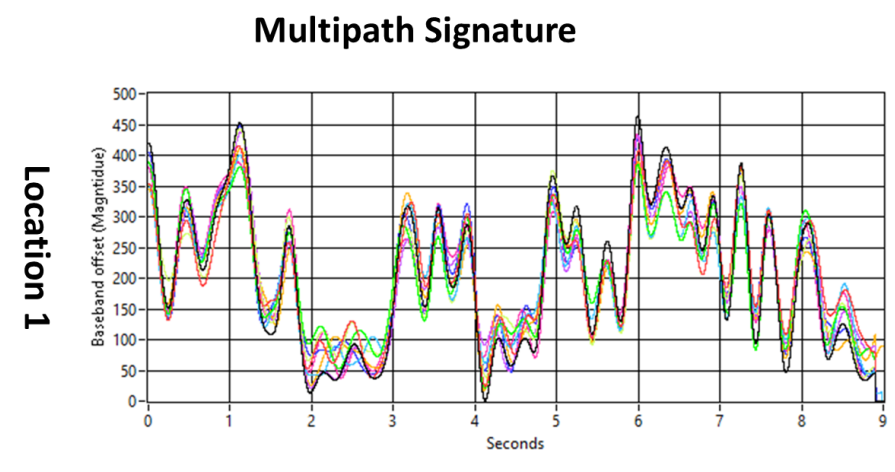

Doppler Signature
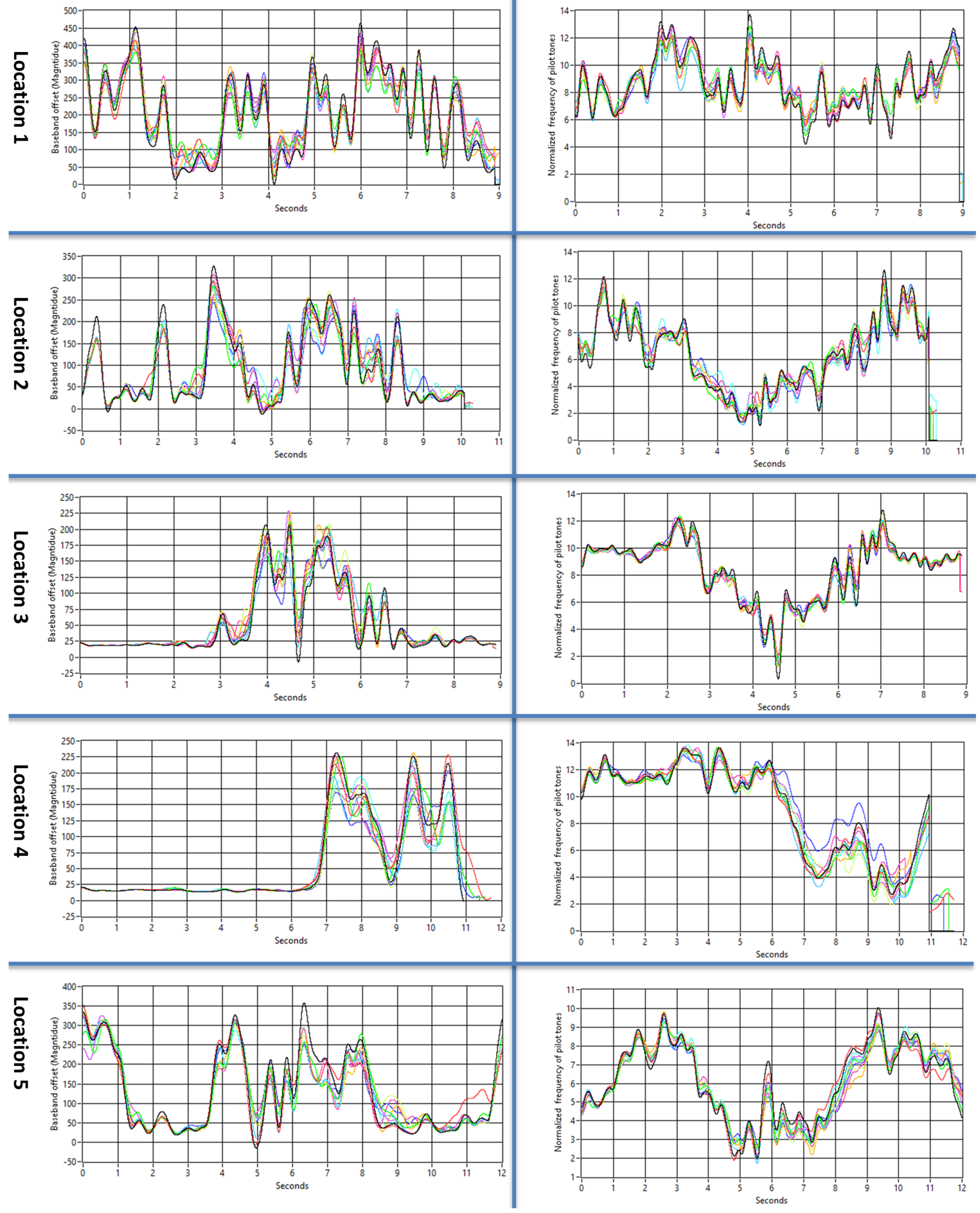

Figure 15: Signatures of one individual in five locations demonstrate that the signatures are stable and location dependent in NLoS case. These graphs provide empirical evidence that the signatures are encoded by room configuration and human gait. The reason for the different signatures at different locations is that multipath channel varies from location to location. 
individuals passed by repeatedly. The broadcasting omnidirectional antenna was placed in the center of the room (NLoS) or hallway $(\mathrm{LoS})$, and the receiving horn antenna was placed at an approximate height of 1.5 meters at the end of the hallway or room facing the test subjects. For the LoS case, the signal capture happened in a hallway, where the individuals were walking repeatedly in front of the horn antenna. The test subjects were told to walk as naturally and consistently as possible. The TX was placed in the room or hallway, simulating a Wi-Fi deployment using a $100 \mathrm{~ms}$ beacon from an omnidirectional antenna. The RF acquisition was aligned to the start and stop times, such that recorded time determined the location of the individuals.

\subsection{Baseline: Control Case Signature}

The challenge addressed in this section is the stability of the baseline signature with no movement. The control case was a hallway of an office and room devoid of movement. As expected, both signatures remained flat, because there was no movement to cause Doppler shift or multipathing (Figure 16).
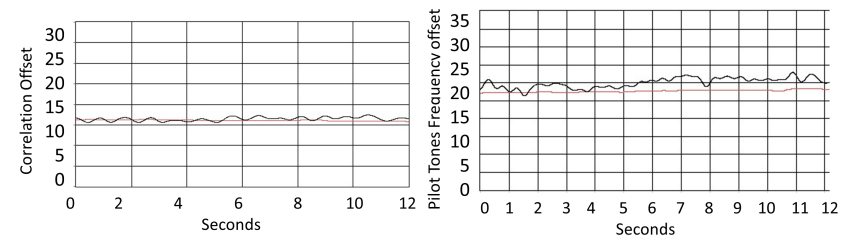

Figure 16: LoS (black) and NLoS (red) flat baselines demonstrate multipath and Doppler features with no movement.

\subsection{Repeatability and Uniqueness}

The experiments demonstrated the uniqueness and repeatability of the signature. Seven individuals walked the same area at least five times. The signature graphs' different colored lines, in each line-of-sight and behind-wall cases, show that the structures of the graphs are similar and make good signatures. Figure 14 shows the signatures from seven individuals in both LoS and NLoS cases. These signature graphs demonstrated that the signatures are unique for each person and repeatable. Signatures for the persons are dependent on the antennas' locations and room configuration, which, if changed, would require recalibration due to new signatures. However in most homes, we can reasonably assume that furniture movement happens infrequently. In most Wi-Fi network deployments, router locations remain fixed.

\subsection{Location Signature Uniqueness}

Figure 15 shows unique signatures for the same person at five different locations in NLoS. The signature was acquired from multiple locations in the same room. The antenna was placed at varying heights, ranging from one to three meters from the ground, at various angles. Based on the signatures, we can reasonably conclude that the signatures are encoded by the location and room configuration. Wobly can safely preserve privacy by encoding multipath and Doppler Effect from gaits and room configurations. Different antenna placements and room configurations cause different multipath channels, and thus produce different signatures.
Table 1: Naïve Bayes classifying features sets for LoS (Top) and NLoS (Bottom) with Male (M) and Female (F) cases

$\begin{array}{llllllll}\text { LOS } & \text { P1 } & \text { P2 } & \text { P3 } & \text { P4 } & \text { P5 } & \text { P6 } & \text { P7 } \\ \text { NLOS } & \text { M } & \text { M } & \text { M } & \text { F } & \text { F } & \text { F } & \text { F } \\ \text { P1 } & 89 \% & 3 \% & 3 \% & 2 \% & 2 \% & 1 \% & 1 \% \\ \text { M } & 83 \% & 5 \% & 4 \% & 1 \% & 4 \% & 3 \% & 3 \% \\ \text { P2 } & 3 \% & 83 \% & 8 \% & 2 \% & 2 \% & 2 \% & 3 \% \\ \text { M } & 6 \% & 78 \% & 5 \% & 4 \% & 5 \% & 2 \% & 2 \% \\ \text { P3 } & 6 \% & 1 \% & 85 \% & 2 \% & 4 \% & 2 \% & 2 \% \\ \text { M } & 8 \% & 3 \% & 81 \% & 3 \% & 2 \% & 2 \% & 2 \% \\ \text { P4 } & 1 \% & 2 \% & 1 \% & 88 \% & 5 \% & 3 \% & 1 \% \\ \text { F } & 6 \% & 3 \% & 4 \% & 70 \% & 9 \% & 8 \% & 3 \% \\ \text { P5 } & 1 \% & 1 \% & 2 \% & 7 \% & 85 \% & 4 \% & 1 \% \\ \text { F } & 4 \% & 2 \% & 3 \% & 6 \% & 74 \% & 7 \% & 4 \% \\ \text { P6 } & 1 \% & 1 \% & 1 \% & 2 \% & 4 \% & 91 \% & 4 \% \\ \text { F } & 3 \% & 7 \% & 4 \% & 6 \% & 6 \% & 74 \% & 6 \% \\ \text { P7 } & 1 \% & 2 \% & 1 \% & 2 \% & 2 \% & 4 \% & 88 \% \\ \text { F } & 3 \% & 3 \% & 2 \% & 4 \% & 2 \% & 6 \% & 80 \%\end{array}$

\subsection{Feature differentiability}

The challenge addressed in this section is differentiation among the feature sets. Extracting features and plotting the two feature vectors against each other, sorted by closest pulse count, the clustering diagrams visualize the differentiability of the feature sets. Figure 17 shows that the classifiers can produce enough distinction between Seven persons in both line-of-sight and non-line-of-sight cases. This clustering diagram does not include packet count or timing information.
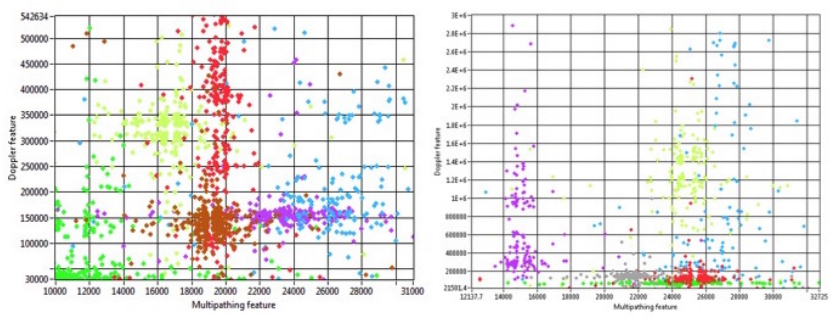

Figure 17: Clustering diagram of gait features demonstrates differentiation with the two feature sets aligned by time.

When removing packets that had little Doppler or multipath interference and adding packet count to the feature set, the simple naïve Bayes classifier was correct $87 \%$ with line-of-sight and $77 \%$ with behind-wall (Table 1).

\section{RELATED WORK}

This section describes previous studies addressing the challenges of monitoring humans to perform actions and modeling human behaviors by tracking body parts, identifying persons, and localizing individuals [29]. Sensors currently used include RF antennas, cameras, capacitors, accelerometers, and ultrasonic detectors. The following sections are previous work categorized by image capture (sections 6.1) and RF (section 6.2). All reviewed works of RF have failed to identify and localize individuals based on intrinsic body movement.

Unlike previous research, this paper profiles the human gait by encoding multipathing and Doppler Effect to cre- 
ate signatures for biometrics, enabling tracking and tagging. This technique uses existing Wi-Fi signals utilizing only a receiver SDR system with through-wall penetration.

\subsection{Imaging Capture and Processing}

This section discusses recent research for human identification using computer vision. Traditional image capture systems require direct LoS or special thermal cameras. These systems are expensive in terms of installation and computational needs. Cameras raise issues with privacy and information assurance, as those devices are easily hacked.

Leveraging computation power of the cloud, eyewear can detect subjects using facial recognition [35]. InSight provides identification of individuals without facial recognition by color pattern and behavior matching [33]. PIXEL enables light based positioning systems using light based modulation using a low resolution camera [40].

\subsection{RF and Wireless system}

This section discusses previous research done to sense human movements using RF (section 6.2.1) and RF based localization techniques (section 6.2.2). RF allows for NLoS and lower cost architecture. All reviewed research has not been shown to produce biometrics on individuals' gait.

\subsubsection{Using RF Waves to Sense Persons or Body Parts}

Recent research used human signals detected by RF sensors have provided the possibility of automation. These systems had the ability to penetrate walls, track body parts, and sense multiple persons' locations [24]. Channel State Information (CSI) based human Activity Recognition and Monitoring $(C A R M)$ system identifies human body parts speed and specific activity. WiDraw uses Wi-Fi signals' Angle-of-Arrival to track hand trajectories to an error rate lower than five $\mathrm{cm}$. WiDraw achieved recognition of letters, words, and sentences to an accuracy rate of $91 \%$ [30]. By using inaudible sound pulses, AAMouse can turn a human hand into a mouse. AAMouse measures both sound frequency shift and the phase of the Wi-Fi signal to track the device at an error rate of $1.4 \mathrm{~cm}$ [42]. WiTrack uses Time-of-Flight from multiple antennas to locate individuals through walls [1], and WiTrack2.0 introduces Successive Silhouette Cancellation to localize multiple persons [3]. E-Gesture is an energy efficient gesture recognition system which uses motion sensing and smart phones [25]. These systems did not provide biometrics or specific person signature detection, but they are focused on localization and detecting movements.

\subsubsection{Using Electromagnetic Waves to Localize}

Tracking devices and individuals have been studied heavily using Signal Strength (SS), Time Difference of Arrival (TDoA), and Angle of Arrival (AoA) [22]. These techniques always use some sort of statistical algorithm with geometry to determine location. SpotOn uses CSI and applies algorithms to estimate the direction and triangulation from multiple Wi-Fi access points. SpotOn achieved accuracy of $0.6 \mathrm{~m}$ in LoS and $1.5 \mathrm{~m}$ in NLoS cases [17]. Tadar tracks moving objects using Commercial of the Self RFID readers and tags in 2D plane, converting RFIDs into antenna arrays for through-wall detection [38]. RFID receivers and transmitters placements optimization were studied to improve accuracy [32]. FollowME uses geomagnetic field and natural walking patterns from previous users for navigation in indoor and semi-outdoor scenarios [28]. APIT is a range free solution for random nodes with irregular radio patterns [12]. SpinLoc is able to provide precise indoor localization using Doppler Effect [7]. A distributed online learning algorithm was able to converge on the location using a wireless sensor network [11].

\section{FUTURE WORK}

For Wobly to function in the real-world, it must track multiple persons and adapt to room changes. WiTrack2.0 [3] has already studied tracking multiple persons and limbs. For multiple room configuration cases, the two main methods are empirical-based machine learning and propagation scattering inversion. Machine learning requires calibration, and scattering inversion requires numeric solutions to large amount of non-linear Maxwell equations. Techniques like deep-learning should also fit the changing scattered field to human movement, resulting in fine location information. Both techniques should model noise from weather or other interferences. We recognize that the current empirical data is limited, requiring more diverse testing subjects, but Wobly extends existing developed gait-based biometric analysis.

\section{CONCLUSIONS}

We present Wobly, a SDR system, that encoded individuals' gaits and room configuration by reflecting Wi-Fi signals off of individuals. The encoded signatures preserved privacy through the uniqueness of how individuals move and the channel interference. We presented 1) methods of tracking individuals in LoS and NLoS, showing uniqueness of signatures, which is encoded by gaits and room configurations; 2) signal processing techniques to measure human bodies' Doppler shift and multipathing creating biometric signatures; and 3) empirical analysis to differentiate people walking in LoS and NLoS. Using wavelets, Wobly characterized the signatures providing feature vectors for a Naïve Bayes Classifier. A clustering visualization utilized the two feature vectors and demonstrated that individual gaits are differentiable. A simple Naïve Bayes classifiers identified persons at $87 \%$ and $77 \%$ correctness in LoS and NLoS cases respectively. Using Wi-Fi signals, this method penetrated a wall and did not require additional attached devices or special signals.

\section{ACKNOWLEDGEMENT}

This work is supported by NSF grants CNS-1503590 and CNS-1539047.

\section{REFERENCES}

[1] Adib, K. Fadel, K. Zach, M. Dina, and R. C. 3d tracking via body radio reflections. NSDI, 2014.

[2] K. Adib. See through walls with wi-fi! SIGCOMM, 2013.

[3] K. Adib, Kabelac. Multi-person localization via rf body reflections. NSDI, 2015.

[4] Brown. The intrinsic factors in the act of progression in the mammal. PRSLBS, 1911.

[5] L. Cai, S. Machiraju, and H. Chen. Defending against sensor-sniffing attacks on mobile phones. In MobiHeld, pages 31-36. ACM, 2009. 
[6] G. Carullo, A. Castiglione, G. Cattaneo, A. De Santis, U. Fiore, and F. Palmieri. Feeltrust: Providing trustworthy communications in ubiquitous mobile environment. In AINA, 2013.

[7] H.-l. Chang, J.-b. Tian, T.-T. Lai, H.-H. Chu, and P. Huang. Spinning beacons for precise indoor localization. In Sensys, pages 127-140. ACM, 2008.

[8] S. Dawson-Haggerty, A. Krioukov, J. Taneja, S. Karandikar, G. Fierro, N. Kitaev, and D. E. Culler. Boss: Building operating system services. In NSDI, volume 13, pages 443-458, 2013.

[9] L. Du, Kibbe. Improved peak detection in mass spectrum by incorporating continuous wavelet transform-based pattern matching. Bioinformatics, 2006.

[10] S. Foresti. Preserving privacy in data outsourcing, volume 99. Springer Science \& Business Media, 2010.

[11] A. Galstyan, B. Krishnamachari, K. Lerman, and S. Pattem. Distributed online localization in sensor networks using a moving target. In IPSN, pages 61-70. IEEE, 2004.

[12] T. He, C. Huang, B. M. Blum, J. A. Stankovic, and T. Abdelzaher. Range-free localization schemes for large scale sensor networks. In MobiCom, pages 81-95. ACM, 2003.

[13] Hnat, E. Timothy W, Griffiths, Dawson, and K. Ray, Whitehouse. Doorjamb: unobtrusive room-level tracking of people in homes using doorway sensors. Sensys, pages 309-322, 2012.

[14] J. Hsu, P. Mohan, X. Jiang, J. Ortiz, S. Shankar, S. Dawson-Haggerty, and D. Culler. Hbci: human-building-computer interaction. In BuildSys, pages 55-60. ACM, 2010.

[15] K. L. Huang, S. S. Kanhere, and W. Hu. Preserving privacy in participatory sensing systems. Computer Communications, 33(11):1266-1280, 2010.

[16] H. Iwama, D. Muramatsu, Y. Makihara, and Y. Yagi. Gait-based person-verification system for forensics. In BTAS, 2012.

[17] M. Kotaru, K. Joshi, D. Bharadia, and S. Katti. Spoton: Indoor localization using commercial off-the-shelf wifi nics. IPSN, 2015.

[18] Larsen. Lost that skip in your step? gait is linked to cognitive decline and alzheimer's, 2012.

[19] H. Leung-Yan-Cheong. The gaussian wire-tap channel. IEEE Transactions on Information Theory, 24, 1978.

[20] J. Li, M. H. Au, W. Susilo, D. Xie, and K. Ren. Attribute-based signature and its applications. In CCS, 2010.

[21] A. Luong, S. Madsen, M. Empey, and N. Patwari. Rubreathing: non-contact real time respiratory rate monitoring system. In IPSN, page 412. ACM, 2015.

[22] D. Lymberopoulos, J. Liu, X. Yang, R. R. Choudhury, V. Handziski, and S. Sen. A realistic evaluation and comparison of indoor location technologies: experiences and lessons learned. In IPSN, pages 178-189. ACM, 2015.

[23] A. Lyonnet, Ioana. Human gait classification using microdoppler time-frequency signal representations. IEEE International Radar Conference, 2010.
[24] F. J. Oppermann, C. A. Boano, and K. Römer. A decade of wireless sensing applications: Survey and taxonomy. In The Art of Wireless Sensor Networks, pages 11-50. Springer, 2014.

[25] T. Park, J. Lee, I. Hwang, C. Yoo, L. Nachman, and J. Song. E-gesture: a collaborative architecture for energy-efficient gesture recognition with hand-worn sensor and mobile devices. In SenSys, pages 260-273. ACM, 2011.

[26] Pu, G. Qifan, G. Sidhant, P. Shyamnath, and Shwetak. Whole-home gesture recognition using wireless signals. MobiCom, 2013.

[27] Ranjan, Y. Juhi, and K. Yu, Whitehouse. An rf doormat for tracking people's room locations. UbiComp, 2013.

[28] Shu, S. Yuanchao, H. Kang G, C. Tian, and Jiming. Last-mile navigation using smartphones. MobiCom, 2015.

[29] J. Stankovic. Research directions for the internet of things. Io TJ, 1(1):3-9, 2014.

[30] L. Sun, S. Sen, D. Koutsonikolas, and K.-H. Kim. Widraw: Enabling hands-free drawing in the air on commodity wifi devices. MobiCom, 2015.

[31] A. Varshney, T. Voigt, and L. Mottola. Directional transmissions and receptions for high throughput burst forwarding. In SenSys, page 50. ACM, 2013.

[32] S. Wagner, M. Handte, M. Zuniga, and P. J. Marrón. On optimal tag placement for indoor localization. In PerCom, pages 162-170. IEEE, 2012.

[33] H. Wang, X. Bao, R. Roy Choudhury, and S. Nelakuditi. Visually fingerprinting humans without face recognition. MobiSys, 2015.

[34] W. Wang, A. X. Liu, M. Shahzad, K. Ling, and S. Lu. Understanding and modeling of wifi signal based human activity recognition. MobiCom, 2015.

[35] X. Wang, X. Zhao, V. Prakash, Z. Gao, T. Feng, O. Gnawali, and W. Shi. Person-of-interest detection system using cloud-supported computerized-eyewear. In HSTn, pages 658-663. IEEE, 2013.

[36] Z. Wei. mtrack: High-precision passive tracking using millimeter wave radios. MobiCom, 2015.

[37] J. Xiong, Sundaresan. Tonetrack: Leveraging frequency-agile radios for time-based indoor wireless localizatio. MobiCom, 2015.

[38] L. Yang, Q. Lin, X. Li, T. Liu, and Y. Liu. See through walls with cots rfid system! MobiCom, 2015.

[39] P. Yang, Z. Cao, and X. Dong. Fuzzy identity based signature. IACR Cryptology ePrint Archive, 2008:2, 2008.

[40] Z. Yang, Z. Wang, J. Zhang, C. Huang, and Q. Zhang. Wearables can afford: Light-weight indoor positioning with visible light. MobiSys, 2015.

[41] Yanzi, Y. Zhu, B. Y. Zhao, and H. Zheng. Reusing $60 \mathrm{ghz}$ radios for mobile radar imaging. MobiCom, 2015.

[42] Q. Yun, Chen. Turning a mobile device into a mouse in the air. Mobisys, 2015. 